\title{
Persuasive Discourse Impairments in Traumatic Brain Injury
}

\author{
Zahra Ghayoumi ${ }^{1}$; Fariba Yadegari ${ }^{1,}$; Behrooz Mahmoodi-Bakhtiari ${ }^{2}$; Esmaeil Fakharian ${ }^{3}$; \\ Mehdi Rahgozar ${ }^{4}$; Maryam Rasouli ${ }^{5}$ \\ ${ }^{1}$ Department of Speech Therapy, University of Social Welfare and Rehabilitation, Tehran, IR Iran \\ ${ }^{2}$ Department of Performing Arts, University of Tehran, Tehran, IR Iran \\ ${ }^{3}$ Trauma Research Center, Kashan University of Medical Sciences, Kashan, IR Iran \\ ${ }^{4}$ Department of Biostatistics and Computer, University of Social Welfare and Rehabilitation, Tehran, IR Iran \\ ${ }^{5}$ Department of Pediatrics, Faculty of Nursing and Midwifery, Shahid Beheshti University of Medical Sciences, Tehran, IR Iran \\ ${ }^{*}$ Corresponding author: Fariba Yadegari, Department of Speech Therapy, University of Social Welfare and Rehabilitation, Tehran, IR Iran. Tel: +98-2122180043, Fax: +98-2122180043, \\ E-mail: fa.yadegari@uswr.ac.ir
}

Received: June 25, 2014; Revised: November 8, 2014; Accepted: February 3, 2015

\begin{abstract}
Background: Considering the cognitive and linguistic complexity of discourse production, it is expected that individuals with traumatic brain injury (TBI) should face difficulties in this task. Therefore, clinical examination of discourse has become a useful tool for studying and assessment of communication skills of people suffering from TBI. Among different genres of discourse, persuasive discourse is considered as a more cognitively demanding task. However, little is known about persuasive discourse in individuals suffering from TBI.

Objectives: The purpose of this study was to evaluate the performance of adults with TBI on a task of spoken persuasive discourse to determine the impaired linguistic measures.

Patients and Methods: Thirteen TBI nonaphasic Persian speaking individuals, ranged between 19 to 40 years (Mean $=25.64$ years; SD = 6.10) and 59 healthy adults matched by age, were asked to perform the persuasive discourse task. The task included asking the participants to express their opinion on a topic, and after the analysis of the produced discourse, the two groups were compared on the basis of their language productivity, sentential complexity, maze ratio and cohesion ratio.

Results: The TBI group produced discourses with less productivity, sentential complexity, cohesion ratio and more maze ratio compared the control group.

Conclusions: As it is important to consider acquired communication disorders particularly discourse impairment of brain injured patients along with their other clinical impairments and regarding the fact that persuasive discourse is crucial in academic and social situations, the persuasive discourse task presented in this study could be a useful tool for speech therapists, intending to evaluate communication disorders in patients with TBI.
\end{abstract}

Keywords:Brain Injuries; Communication Disorders; Linguistics

\section{Background}

Brain injury is the third most common cause of mortality in the world (1). In Iran, road-traffic crashes, which is the major source of brain injuries- cause disability or injury of more than 300,000 persons each year (2).

In recent years, the importance of communication abilities for the long-term quality of life of survivors of TBI has begun to be recognized (3). Deficits in communication skills following TBI are also common. Although motor speech impairment and aphasia can be present, the most frequent problems relate to impairment in cognitive processes is subserving language and communication functions (4). These cognitive-communication impairments include disorganized and tangential discourse. As a consequence, the discourse analysis provides the opportunity for clinicians and researchers to control the interaction among several processing levels and to assess the communicative skills in a more ecological setting. From this perspective, the multi-level procedure for discourse analysis has also interesting theoretical implications, as it may provide insights on the interrelations between the various levels of linguistic processing (micro-linguistic, macro-structural and macro-linguistic analysis, as explained in the following) (5).

Therefore, the clinical examination of discourse has gained interest as a useful tool for studying communication skills after TBI. Discourse is more complex or higher level than those behaviors, which can usually be assessed by means of an aphasia battery reliably (4).

The presence of a frontal lesion following a TBI is common (6); although other lobes lesions like temporal and occipital may be also involved (see (7-9)). The TBI-related behavioral consequences are usually due to damages in the frontal lobes - the lobes, which are employed in the execution, planning, and control of many behaviors 
Ghayoumi Z et al.

such as language functioning - that aphasia tests do not assess those aspects of language ability (10). Therefore, performance examination by means of such tests might show that communicative skills in these patients are intact. However, the listener still has the feeling that their discourses are disorganized, tangential or even off-target, when they are engaged in an interaction. In other words, the language of TBI patients damaged beyond the level of single words or sentences, which such batteries assessed them. Thus, these measures are not sufficiently challenging to illuminate the full nature and extent of cognitive communication impairments in TBIs (4) and reliance on aphasia or traditional language test batteries is not adequate (11-13). Typically, by these tests, their language deficits go undetected or are incompletely delineated (13).

In recent years, researchers have focused on communication after TBI is fixed on abilities in discourse (14). Unlike responding to single items on highly structured language assessment tests, production of discourse requires the speaker to integrate both cognitive and linguistic abilities within a framework of organizing overall thought and social interaction (11).

It is suggested that proficiency in discourse (comprehension and/or production) involves a complex interaction of linguistic and cognitive organizational processes (4). It has been proposed that executive functions may be an important contributor to discourse generation, regardless of the nature of the injury (15). There are a number of discourse genres that can be used in clinical settings. These include, descriptive, narrative, expository, persuasive and conversational discourse (16). Although impaired discourse is the hallmark of posttraumatic brain injury but the most studied genre of discourse produced by patients with TBI is narrative discourse $(13,17)$.

In a research done by Wilson and Proctor, analysis indicated that patients with closed head injury (CHI) used less number of words to express their ideas in writing (18). Coelho, in a cohort study, considered 55 consecutive nonaphasic TBI participants on a task of storytelling. In the narrative of these individuals, more extraneous propositional content were introduced, suggesting problems in the organization of information at the between-sentence level (17). Narrative discourse of 14 severe TBI speakers, who were not aphasic, and a group of neurologically intact participants of size 14 were studied by Marini (19). The group of individuals with TBI represented normal grammatical and lexical skills. However, these patients produced narratives with more cohesion and coherence errors due to the frequent pause in their ongoing utterances, extraneous utterances and derailments, which made their discourse ambiguous and vague.

One factor that should be considered during the evaluation of discourse production is the genre that is assessed (20). Differences between the communication of normal adults and TBI patients may differ depending on genre that is assessed; except for narrative genre, that there are very few studies in other genres in clinical setting. In a study in the domain of expository discourse Hay and Moran investigated expository discourse abilities of nine children with $\mathrm{CHI}$. The children with $\mathrm{CHI}$ differed significantly from their age matched peers across language and information domains and in their capability to formulate an aim or moral in the expository retellings. In addition, differences across genre were found with performance on narrative tasks superior to performance on expository tasks (21).

Persuasive discourse is an area of discourse, which is of particular interest following TBI (20). Persuasion is a complex and essential skill, which continues to develop into early adulthood (22). In general, comparing to narrative production, persuasion task is considered to be more cognitively demanding (23). Persuasive discourse attempts to express an opinion and gives reasons to support that opinion (16). This type of discourse may occur in both formal situations (e.g. school debates, school essays) and informal situations (e.g. convincing a friend to see a movie or urging parents to purchase the latest electronic gadget). The ability to persuade and use arguments effectively is considered a fundamental social interaction skill(24).

Given the cognitive and linguistic sophistication requirements, it is expected that individuals with TBI would show difficulties in persuasive discourse production. However, little is known about persuasiveness in individuals, who have suffered some sort of cognitive or linguistic impairments. One clinical population that may be susceptible to deficits in persuasive discourse is the traumatic brain injured population (20). But to date, there is a lack of study in the domain of persuasive discourse in adults with TBI.

Moran et al. investigated adolescents with acquired brain injury (ABI) to measure their performance in a spoken persuasive discourse task in eight $\mathrm{ABI}$ patients and eight controls, matched for education, gender and age. The two groups were compared on measures of discourse productivity, language content and syntactic complexity. On measures of language productivity and syntactic complexity, there was no statistically significant group difference. But they found significant differences in language content (20).

Depending on the task used for elicitation of discourse and the aim of the analysis, the specific analysis may be chosen at different levels. For example, discourse analysis could be classified into these three basic levels, as in $(25,26)$ :

- Micro-linguistic analysis: Micro-linguistic or withinsentence analyses typically include measures of productivity, verbal errors, propositions, sentential complexity and content units.

- Micro-structural analysis: Across-sentence or microstructural analyses include measures of cohesion and cohesive adequacy. 
Ghayoumi Z et al.

- Macro-structural analysis: In this level, analyses typically involve measures of local and global coherence, which are considered as indicators of thematic unity in a discourse.

\section{Objectives}

This study aimed to examine the performance of persons with TBI during a spoken persuasive discourse task and determine impaired linguistic measures in microlinguistic and microstructural levels of persuasive discourse in TBI patients compared to normal group.

\section{Patients and Methods}

\subsection{Participants}

Thirteen adult survivors of TBI participated in the research. All participants were male. Generally, males are uniformly at higher risk of TBI than females, with the highest male-to-female (M/F) ratios typically occurring in adolescence and young adulthood. In the national sample U.S. ED studies, the M/F ratio was 1.5:1 and 1.7:1(27). This ratio in Iran is even higher: 4.25:1 (28). and ranged in age from 19 to 40 years old $(M=25.64$ years; $S D=6.10)$. To be included in the experimental group, the patients had to be in the phase of neurological stability (19) (months post-onset - mean: 25.75; SD: 36.21; range: 1-120) and do not demonstrate any significant deficits on traditional clinical language tests (29). To evaluate this latter condition, the Aphasia Quotient of the Western Aphasia Battery (WAB), adopted to Persian (30), was performed. None of the TBI participants were aphasic as established through their performance on this test.

Moreover, all were at or above 23 in the adopted version of minimental state examination (MMSE) in Persian (31) when they participated in this research, ensuring that they were oriented to self, environment and place and could answer to simple questions. The mean score of their Glasgow coma scale (GCS) in the acute phase had been 8.14 (nine of participants have severe TBI, GSC score less than 9, and five were moderate with the GCS score between 9 and 12. Participants with mild TBI were excluded from the study). Also, no visual and hearing impairment has been reported for any of the subjects. Participants' educational achievement ranged from 3 to 18 years $(\mathrm{M}=$ 10.08 years; $S D=4.49$ ). All were Persian native speakers and none of them had any history of neurological or psychiatric problem except those related with TBI.

The control group was formed from 59 neurologically intact subjects, 20 males and 39 females ranged in age from 19 to 40 years $(M=25.88$ years; $S D=5.70)$ and all were fluent speakers of Persian. Regarding the gender, as showed by Ghayoumi (29), the discourse of two genders does not have any significant different and considering the control group in our study, the same results hold. In this study, we excluded control subjects who had any his- tory of cognitive impairments, neurological disorders (like stroke, epilepsy or TBI), blindness, deafness or major physical disabilities. Educational achievement in the control group ranged from 11 to 18 years $(M=14.58$ years; $S D=$ 1.99) and their age did not differ significantly from those with TBI $(\mathrm{P}=0.00)$.

Table 1. Demographic and Clinical Characteristics of Subjects With TBI ${ }^{\text {a }}$

\begin{tabular}{lcc}
\hline & Rang & Mean \pm SD \\
\hline Time after injury, $\mathbf{m}$ & $1-120$ & $27.57 \pm 37.01$ \\
Coma duration, $\mathbf{d}$ & $0-270$ & $56.15 \pm 89.04$ \\
GCS, score & $4-13$ & $8.23 \pm 3.46$ \\
MMSE, score & $23-30$ & $26.77 \pm 2.58$ \\
\hline WAB, quotient & $91-100$ & $94.15 \pm 2.73$ \\
\hline
\end{tabular}

a Abbreviations: GCS, Glasgow coma scale; MMSE, minimental state examination; WAB, Western aphasia battery.

\subsection{Procedures}

All participants met personally with the examiner to accomplish a persuasive task where no time limit was imposed, and all sessions were digitally recorded for later analysis.

Following a brief introduction and explanation of the session, two standardized neurophysiological tests were administered: WAB and MMSE. The WAB was administered to insure absence aphasia in patients. The Western aphasia battery is an instrument for assessing the language function of adults, able to discern the presence, degree, and type of aphasia. If subject scores 91 or more, based on this test, he is not considered an aphasic patient (30).

The MMSE test was performed for evaluating the cognitive abilities of all participants to ensure that the presence of residual deficits in cognition did not preclude meaningful participation including the capacity to attend to tasks at hand, comprehend and follow instructions. It is a brief 30-point questionnaire test that is used to screen for cognitive impairment. The maximum score is 30. Cognitive impairment is screened by scores of lower than 23 (31).

Next, the assessment of persuasive abilities was performed using a question and participants were asked to offer an opinion about whether it is better to use the public transport or private vehicle and why. As topic knowledge is likely to affect the quality of persuasive argumentation, this topic was chosen, because it seems to have a widespread appeal and thus, increasing the likelihood that the participants would have personal experiences and ideas about it. For feedback, the examiner responded only with encouragement to proceed the discourse (like "Anything else?") and natural conversational acknowledgements. A discourse was completed when the subject expressed that he/she had nothing more to add. All ses- 
sions were similar in their format. Signing of the consent form occurred at the beginning of the session and the procedure had been previously approved by the ethic committee of the university.

\subsection{Linguistic Measures}

\subsubsection{Productivity}

Productivity is measured according to the amount of produced spoken or written discourse. In this study, the number of C-units per discourse was considered as productivity (18).

\subsubsection{Sentential Complexity}

Sentences may be complex due to various reasons: they can convey complicated ideas, contain infrequent words, or have a complex syntactic structure (32). The focus of this study is placed on syntactic structures, and sentential complexity is defined as the total number of dependent clauses and independent clauses divided by total number of C-units (20).

\subsubsection{Maze}

Maze is defined as a series of words, initial parts of words, or unattached fragments, which does not convey the meaning for the ongoing flow of discourse. By removing the mazes from an utterance, the remaining phrase constitutes a meaningful communication unit. Normal disfluencies, such as filled pauses (ex. "um, uh, I saw it") and revisions (ex. "The girl, I mean, the lady left") occur to some extent in the speech of all individuals. Mazes are manifested in the speech of individuals when expressing an idea that is abstract, complicated or not yet fully developed (33).

In our study, the followings are regarded as mazes: exact repetitions of phrases/words, revisions or false starts, abandoned utterances, non-linguistic vocalizations (e.g. uh, hmm, oh) and audible pauses. For adjusting mazes for the length of produced discourse, we consider the maze per C-Unit, which was calculated by dividing total number of mazes by total number of C-units in each discourse.

\subsubsection{Cohesion}

Cohesion can be defined as the property that distinguishes a sequence of sentences that form a discourse from a random sequence of sentences. It is a series of lexical, grammatical and other relations which provide links between the various parts of a text. Cohesion analysis may include the frequency of occurrence of any kind of cohesive ties (lexical, reference, conjunctive, substitution/ellipsis) (34).

In this study, we consider measuring cohesion per Cunit in which the cohesion is sum of all cohesive ties of any type in the discourse.

\subsection{Analysis}

The recorded discourses of all participants were transcribed verbatim. At first, our analysis included determining C-units, dependent clauses, independent clauses, mazes and each type of cohesion ties (reference, substitution, ellipses, lexical). Then computation of productivity, sentential complexity, maze ratio and cohesion ratio has been performed.

Following computation of each variable, the persuasive discourse performance of the two studied groups was analyzed by independent t-test (productivity and sentential complexity) and Mann-Whitney U test (for cohesion ratio and maze ratio) to determine significant group differences.

\subsection{Reliability of Analysis}

The reliability of this analysis was calculated in two levels. To measure the intra-rater reliability, about $15 \%$ of samples $(n=10)$ were selected randomly to re-analyze by researcher three weeks after the initial analyses were completed.

In another level, inter-rater reliability of the linguistic analysis was calculated by measuring all variables in a second round by an independent researcher using about $15 \%$ of randomly selected samples $(n=10)$.

Reliability for each variable was calculated by Pearson correlation coefficient. Average intra-rater reliability scores for productivity, sentential complexity, maze and cohesion were $100 \%, 100 \%, 100 \%$ and $99 \%$ (with $\mathrm{P}<0.0001$ for all), respectively, and inter-rater reliability scores for productivity, sentential complexity, maze and cohesion were $99 \%, 100 \%$, 99\% and 98\% correspondingly $(\mathrm{P}<0.001$ for all), respectively.

\section{Results}

Table 2 presents the range, mean and standard deviation of each measured value for both TBI patients and control subjects.

\subsection{Results of Micro-Linguistic}

Table 2 represents the mean values of all microlinguistic measures, including productivity, sentential complexity and maze ratio, for each group. Statistical analysis showed that the TBI participants produced persuasive discourses with significantly less productivity $(\mathrm{P}=0.02)$ and sentential complexity $(\mathrm{P}=0.03)$ compared to the control healthy group. Although individuals with TBI produce more mazes in each $\mathrm{C}$-unit, the difference was not statistically significant $(\mathrm{P}=0.7)$

\subsection{Result of Microstructural}

The mean value of cohesion ratio, as the microstructural measure, is reported in Table 2. The TBI participants produced discourses with significantly less cohesion in each C-unit $(\mathrm{P}=0.04)$. 
Ghayoumi Z et al.

Table 2. Mean, Range and Standard Deviation for Patients With Traumatic Brain Injuries and Healthy Control Subjects on Measured Values $^{\text {a }}$

\begin{tabular}{lcccc}
\hline & \multicolumn{2}{c}{ TBIs } & HC \\
\cline { 2 - 5 } & Range & Mean \pm SD & $2-19$ & Mean \pm SD \\
\hline Productivity & $1-14$ & $6.92 \pm 3.61$ & $0-2.2$ & $9.88 \pm 4.20$ \\
Sentential complexity & $0-1.50$ & $0.38 \pm 0.50$ & $0-2.16$ & $0.666 \pm 0.41$ \\
Maze ratio & $0-4$ & $0.70 \pm 1.17$ & $0.5-3.5$ & $0.54 \pm 0.46$ \\
Cohesion ratio & $0.37-3$ & $1.29 \pm 0.90$ & $1.57 \pm 0.65$ \\
\hline
\end{tabular}

a Abbreviations: HC, Healthy Control; TBI, Traumatic Brain Injury.

\section{Discussion}

\subsection{Linguistic Measures}

The current study examined features of persuasive discourse in a group of adult TBI speakers compared to a healthy controls group. One important objective was to find language measures that are different in two groups. Obtained results indicated that persuasive discourse of persons after TBI differed significantly from those of controls. Note that in this study, the level of education was not completely matched and thus, research results should be interpreted in a cautious manner.

\subsection{Productivity}

An important finding of the current study is that the productivity of persuasive discourse subsequent to TBI reveals impairment across language measures. This finding is in line with the works of Body and Perkins (14), Hay and Moran (21), who demonstrated that the discourse performance of the TBI patients is below the control group (noting that the second study was performed on children). Considering the obtained discourse samples, this was exactly the expected results; as our experiments show that the normal participants try to fulfill the expectations of the persuasive argument by providing reasons and produce more supporting reasons in comparison to TBIs. However, this result is different from findings of Marini et al. (19) and Wilson and Proctor (18). Also, this is in contrast with Moran et al. (20), where authors didn't find significant differences on productivity measures between normal control and TBI adolescents. In the former study, the authors consider different aspects of productivity, some of which were similar between TBI group and normal controls and others (including speech rate and mean length of units) were significantly different between two groups. However, we considered productivity as number of C-units.

\subsection{Sentential Complexity}

Syntactic complexity can be used to assess language proficiency (35). Our TBI patients showed lower sentential complexity compared to normal groups. This indi- cates that the TBI participants produced fewer dependent clauses in each C-unit and therefore have less language proficiency than their typically peers. Consistent with current study, Campbell and Dollaghan (36), found that sentential complexity remained a problem for six of their CHI subjects out of nine. Also, in Glosser and Desir's study, the measures of syntactic errors in CHI group was significantly more compared to normal subjects, but the CHI group was not impaired on syntactic complexity measure (37).

In Moran et al. study (20), no significant difference was found in sentential complexity between the two studied groups (normal and TBI). The disparity in sentential performance among studies might be due to differences between the types of scaling complexity or task differences.

\subsection{Maze}

In our study, TBI patients have produced more maze (per C-unit) than the control group. Fagan reported that mazes could be interpreted as a factor of language planning and could be noted at specific grammatical points within utterances, for example, connecting ideas (38). Moreover, they can be considered as indications of the verbal decision-making behavior (18). According to the current findings, the use of more mazes could be due to participants needing to spend more time planning as they have not been given examples to direct their ideas. Also, in Wilson and Proctor's study that investigated story generation in written discourse, CHI patients produced more mazes than controls, but the difference was not significant (18).

\subsection{Cohesion}

Measures of cohesion addressed to determine whether participants connect and link their utterances and ideas in a logical manner for a listener to follow. The concept of cohesion proposed by Halliday and Hasan (34) refers to the semantic and grammatical connections between the segments of a text or discourse. In the current study, TBI participants showed significantly less cohesive ties (per Cunit) than control group. This shows that TBI participants have problem in connecting and linking their utterances and ideas in a logical manner for a listener to follow. 
In a similar study, Marini showed TBI participants demonstrated significantly more cohesion errors $(19,39)$. Also, Mentis and Prutting noticed that compared to normal subjects, their $\mathrm{CHI}$ participants used fewer cohesive ties in their narrative tasks (40). But Glosser and Deser noted that their CHI subjects did not differ from the normal people on their cohesion measures (41). Hence, considering discourse tasks from children and adults with TBI in different studies have yielded inconsistent findings (42).

\subsection{Conclusions}

The current research arose from a clinical demand to obtain materials, which may be used for the evaluation of communication skills after TBI. The persuasive discourse tasks presented in this study may be useful for clinicians, aiming to evaluate communication disorders in persons with TBI. Our findings showed that TBI patients demonstrate discourse disorder as a primary clinical symptom and their speeches are impaired on both microlinguistic and microlinguistic measures in comparison with the control group. So, it is important to consider disorder of these subjects along their other clinical impairments.

\section{Acknowledgements}

The authors wish to thank Zahra Babaei and Sedighe Abbasian for their invaluable helps in data collection.

\section{Authors' Contributions}

The work is a part of Zahra Ghayoumi PhD dissertation under the supervision of Dr. Fariba Yadegari. Dr. Behrooz Mahmoodi Bakhtiari was the linguistics advisor of the work, mainly with respect to defining the measures and analyzing the discourse. Dr. Esmaeil Fakharian was the neurological advisor. He evaluated and determined the diagnosis, all TBI patients. Dr. Mehdi Rahgozar and Dr. Maryam Rasouli, were both advisors of the thesis, contributed to the statistical analysis and construction of the test.

\section{Funding/Support}

This study was derived from the PhD thesis of the first author.

\section{References}

1. Shakeri M, Mahdkhah A, Panahi F. S100B Protein as a Post-traumatic Biomarker for Prediction of Brain Death in Association With Patient Outcomes. Arch Trauma Res. 2013;2(2):76-80.

2. Fazel MR, Fakharian E, Mahdian M, Mohammadzadeh M, Salehfard L, Ramezani M. Demographic Profiles of Adult Trauma During a 5 Year Period (2007-2011) in Kashan, IR Iran.Arch Trauma Res. 2012;1(2):63-6.

3. Theodoros DG. Traumatic brain injury: Associated speech, language, and swallowing disorders.: Cengage Learning; 2001.

4. Coelho CA. Management of discourse deficits following traumatic brain injury: progress, caveats, and needs. Semin Speech Lang. 2007;28(2):122-35.

5. Marini A, Andreetta S, del Tin S, Carlomagno S. A multi-level ap- proach to the analysis of narrative language in aphasia. Aphasiology. 2011;25(11):1372-92.

6. Dardier V, Bernicot J, Delanoe A, Vanberten M, Fayada C, Chevignard $\mathrm{M}$, et al. Severe traumatic brain injury, frontal lesions, and social aspects of language use: a study of French-speaking adults. J Commun Disord. 2011;44(3):359-78.

7. Besenski N. Traumatic injuries: imaging of head injuries. Eur Radiol.2002;12(6):1237-52.

8. Bigler ED. Distinguished Neuropsychologist Award Lecture 1999 The lesion(s) in traumatic brain injury: implications for clinical neuropsychology. Arch Clin Neuropsychol. 2001;16(2):95-131.

9. Pierallini A, Pantano P, Fantozzi LM, Bonamini M, Vichi R, Zylberman R, et al. Correlation between MRI findings and long-term outcome in patients with severe brain trauma. Neuroradiology. 2000;42(12):860-7.

10. Biddle KR, McCabe A, Bliss LS. Narrative skills following traumatic brain injury in children and adults. J Commun Disord. 1996;29(6):447-68.

11. Hux K, Wallace S, Evans K, Snell J. Performing cookie theft picture content analyses to delineate cognitive-communication impairments. 2008;16(2).

12. Coelho C, Ylvisaker M, Turkstra LS. Nonstandardized assessment approaches for individuals with traumatic brain injuries. Semin Speech Lang. 2005;26(4):223-41.

13. Coelho CA. Discourse production deficits following traumatic brain injury: A critical review of the recent literature. Aphasiology. 1995;9(5):409-29.

14. Body R, Perkins MR. Validation of linguistic analyses in narrative discourse after traumatic brain injury. Brain Inj. 2004;18(7):707-24 .

15. Douglas JM. Relation of executive functioning to pragmatic outcome following severe traumatic brain injury. J Speech Lang Hear Res. 2010;53(2):365-82

16. Ball MJ, Perkins MR, Müller N, Howard S. The handbook of clinical linguistics.: John Wiley \& Sons; 2009.

17. Coelho CA. Story narratives of adults with closed head injury and non-brain-injured adults: influence of socioeconomic status, elicitation task, and executive functioning. J Speech Lang Hear Res. 2002;45(6):1232-48.

18. Wilson BM, Proctor A. Written discourse of adolescents with closed head injury. Brain Inj. 2002;16(11):1011-24.

19. Marini A, Galetto V,Zampieri E, Vorano L, Zettin M, Carlomagno S. Narrative language in traumatic brain injury. Neuropsychologia. 2011;49(10):2904-10.

20. Moran C, Kirk C, Powell E. Spoken persuasive discourse abilities of adolescents with acquired brain injury. Lang Speech Hear Serv Sch. 2012;43(3):264-75.

21. Hay E, Moran C. Discourse formulation in children with closed head injury. Am J Speech Lang Pathol. 2005;14(4):324-36.

22. Nippold MA, Ward-Lonergan JM, Fanning JL. Persuasive writing in children, adolescents, and adults: a study of syntactic, semantic, and pragmatic development. Lang Speech Hear Serv Sch. 2005;36(2):125-38.

23. Felton M, Kuhn D. The Development of Argumentive Discourse Skill. Discourse Processes. 2001;32(2-3):135-53.

24. Bartsch K, London K, Campbell MD. Children's attention to beliefs in interactive persuasion tasks. Dev Psychol. 2007;43(1):111-20.

25. Davis GA, Coelho CA. Referential cohesion and logical coherence of narration after closed head injury. Brain Lang. 2004;89(3):508-23.

26. Lê K, Mozeiko J, Coelho C. Discourse analyses: Characterizing cognitive-communication disorders following TBI. The ASHA Leader. 2011;16:18-21.

27. Bruns JJ, Hauser WA. The epidemiology of traumatic brain injury: a review. Epilepsia. 2003;44 Suppl 10:2-10.

28. Rahimi-Movaghar V, Saadat S, Rasouli MR, Ghahramani M, Eghbali A. The incidence of traumatic brain injury in Tehran, Iran: a population based study. Am Surg. 2011;77(6):e112-4.

29. Ghayoumi Z, Coelho CA, Grela B, Corso M, Gamble A, Feinn R. Microlinguistic deficits in the narrative discourse of adults with traumatic brain injury. Brain Inj. 2005;19(13):1139-45.

30. Nilipour R, Pourshahbaz A, Ghoreyshi ZS. Reliability and Validity of Bedside Version of Persian WAB. Basic and Clinical. 2014:253.

31. Ansari NN, Naghdi S, Hasson S, Valizadeh L, Jalaie S. Validation of 
a Mini-Mental State Examination (MMSE) for the Persian population: a pilot study. Appl Neuropsychol. 2010;17(3):190-5.

32. Shetreet E, Friedmann N, Hadar U. An fMRI study of syntactic layers: sentential and lexical aspects of embedding. Neuroimage. 2009;48(4):707-16.

33. Use of mazes in the narrative language samples of bilingual and monolingual 4-to 7-year old children. In: Fiestas CF, Bedore LM, Peña ED, Nagy V], Cohen J, McAlister KT, et al editors. . ISB4: Proceedings of the 4th International Symposium on Bilingualism. 2005 pp. $730-40$.

34. Halliday MAK, Hasan R. Cohesion in English.: Routledge; 1976.

35. Lu X. Automatic analysis of syntactic complexity in second language writing. International Journal of Corpus Linguistics. 2010;15(4):474-96.

36. Campbell TF, Dollaghan CA. Expressive language recovery in severely brain-injured children and adolescents. J Speech Hear Dis- ord.1990;55(3):567-81.

37. Glosser G, Deser T. Patterns of discourse production among neurological patients with fluent language disorders. Brain Lang. 1991;40(1):67-88.

38. Fagan WT. The relationship of the" maze" to language planning and production. Research in the Teaching of English. 1982:85-95.

39. Marini A, Zettin M, Galetto V. Cognitive correlates of narrative impairment in moderate traumatic brain injury. Neuropsychologia. 2014;64C:282-8.

40. Mentis M, Prutting CA. Cohesion in the discourse of normal and head-injured adults. J Speech Hear Res. 1987;30(1):88-98.

41. McDonald S. Pragmatic language skills after closed head injury: ability to meet the informational needs of the listener. Brain Lang. 1993;44(1):28-46.

42. McLeod J, Von Treuer K. Towards a Cohesive Theory of Cohesion. International Journal of Business and Social Research. 2013;3(12):1-11. 